\title{
Crohn's disease, tuberculosis or NSAID-induced colopathy: a case report
}

\author{
Deian Jelev', Dimitar Popov', Lubomir Marinchev², \\ Rosen Nikolov', Radina Ivanova ${ }^{1}$, Zahariy \\ Krastev'.
}

1. linic of Gastroenterology, University Hospital St. Ivan Rilsky, Sofia;

2. Rheumatology Department, MHAT SOFIAMED, Sofia;

\begin{abstract}
One very well known to us 70-year-old patient with successful intralesional experimental therapy with interleukin-2 for abdominal mesothelioma (published in 2001) and local recurrence 14 years later, COPD and coronary heart disease, as well as with history of coxarthrosis for several years, was presented to us with an inflammatory syndrome and a slowly progressive anemia improved after the antibiotic treatment, and later development of real iron deficiency anemia. A sacroiliitis was proven by two bone histologies. Although the patient was without gastrointestinal disorders, a colonoscopy was performed and Crohn's disease was established. We discussed: 1) Crohn's disease with involvement of the joint; 2) bone metastasis from mesothelioma; 3) tuberculosis (based on histological evidence of bone granulomas, history of old pulmonary disease, and histology data of intestinal tuberculosis). Later the patient reported intake of NSAIDs for about two years. Despite the negative test, antituberculous (TB) medication was initiated. The conclusions from reviews of the biopsy samples varied from Crohn's disease, ulcerative colitis, tuberculosis to ischemic colitis. Control colonoscopy revealed cicatrices in the large bowel and few small erosions. We applied Methotrexate, despite the risk of recurrence of the mesothelioma. Methotrexate caused an expected lymphopenia. The subcutaneous interleukin-2 in low doses gradually normalized blood cell count and the status has been in control for 3 years.
\end{abstract}

Keywords: Mesothelioma, interleukin-2, methotrexate, sacroiliitis, anemia, colon histology. 


\section{Background}

Intestinal tuberculosis (TBC) and colopathy induced by non-steroidal anti-inflammatory drug (NSAID) can mimic Crohn's disease (CD) and thus can cause diagnostic difficulties $(1,2)$.

Several studies showed that CD and TBC overlap in clinical, endoscopic and histological features (2-4). Sacroiliitis $(\mathrm{SI})$ is one of the most frequent extraintestinal manifestations in patients with inflammatory bowel disease (IBD) and can complicate medical behavior (5). Longitudinal ulcers are typical for CD, but can also be seen in patients with intestinal TBC and NSAID-induced colopathy (1, 2). In addition, some of the gut erosions, ulcers and strictures in CD are very similar to that caused by NSAID (1). Diaphragm strictures are pathognomonic findings of NSAID-induced colopathy, but are very rarely seen (1).

Granulomas are typical histological findings in TBC, but are also present in patients with CD (2). Caseous necrosis is an important histological feature of TBC, but in some studies it was found in only $13.3 \%$ of patients with gut TBC (2). Tuberculous SI is an uncommon variant of joint tuberculosis and mostly case reports are communicated (6).

For all the reasons described above there is a growing number of reports describing instances of misinterpretation of endoscopic and histological findings that caused wrong or delayed diagnosis (7-9). As differential diagnosis in such patients is a real challenge, any presented case is of great clinical importance.

\section{Case Description}

The patient is a 70- year-old male with history of:

- $\quad$ peritoneal mesothelioma in complete remission for 12 years, achieved by experimental therapy with interleukin 2 (IL-2) and recent recidive with minimal ascites. The relevant results of local IL-2 therapy in this patient were previously published (10)

- $\quad$ HLA B27 positive sacroillitis

- $\quad$ right hip joint replacement due to severe coxitis. Residual perijoint infiltrate proven with MRI and US

- $\quad$ COPD and coronary heart disease

- anemia: initially normocytic normochromic ( $\mathrm{Hb} 96 \mathrm{~g} / \mathrm{l})$, tended to improve during antibiotic therapy $(\mathrm{Hb} 110 \mathrm{~g} / \mathrm{l})$, but later became truely iron-deficient $(\mathrm{Hb} 97 \mathrm{~g} / \mathrm{l})$

- $\quad$ inflammatory syndrome (CRP $102 \mathrm{mg} / \mathrm{l})$

The patient was without gastrointestinal disorders. The main complaints in the last 2 years were permanent severe right hip joint and lumbar pain, accompanied with sub-febrile episodes. He received NSAIDs over the last 2 years.

Colonoscopy with biopsies was done due to anemia and the endoscopic findings were interpreted as CDcompatible: spastic segments and strictures, diffuse hyperemia of mucosa from the mid transversum to cecum with large and deep ulcerations (fig.1). A histological examination of colon specimens showed focal distortion of mucosal architecture, crypts with cryptitis and moderate chronic inflammation in lamina 
propria, as well as an ulceration with inflammatory granulation tissue at the bottom and hemorrhages (fig. 2).

Fig. 1. Colonoscopy with an ulcer.

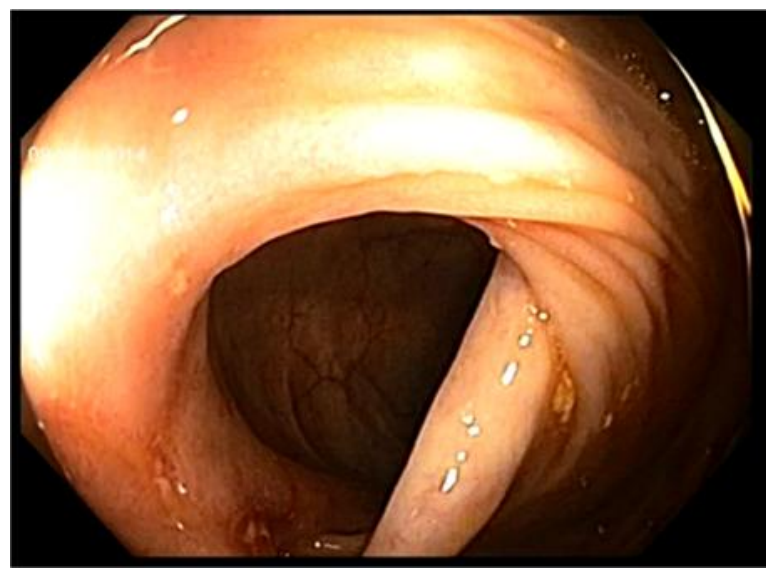

Fig. 2. Colon biopsy specimen, showing inflammatory granulation tissue from the site of cleft-like ulceration (HE, 40x)

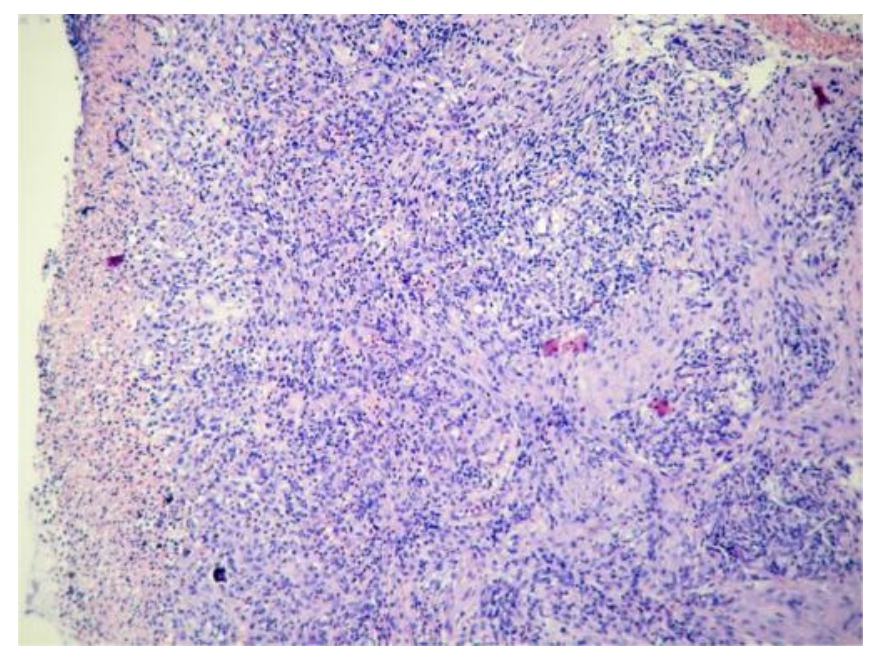

Four different histological conclusions were reached after consequent reviews of the same biopsy samples by independent pathologists: CD; ulcerative colitis (UC); tuberculosis (TBC) and NSAID-induced colopathy. In addition there were 4 different reports from bone and soft tissue biopsies obtained during hip joint replacement: a mesothelioma; tumoral calcinosis, bone necrosis with granulomas type "foreign body" and TBC. The main histological features of the bone specimens were focal necrosis and regeneration, and also some granulomas types of foreign body (fig. 3). 
Fig. 3. Bone biopsy, showing areas with necrosis and regeneration (HE, x 40)

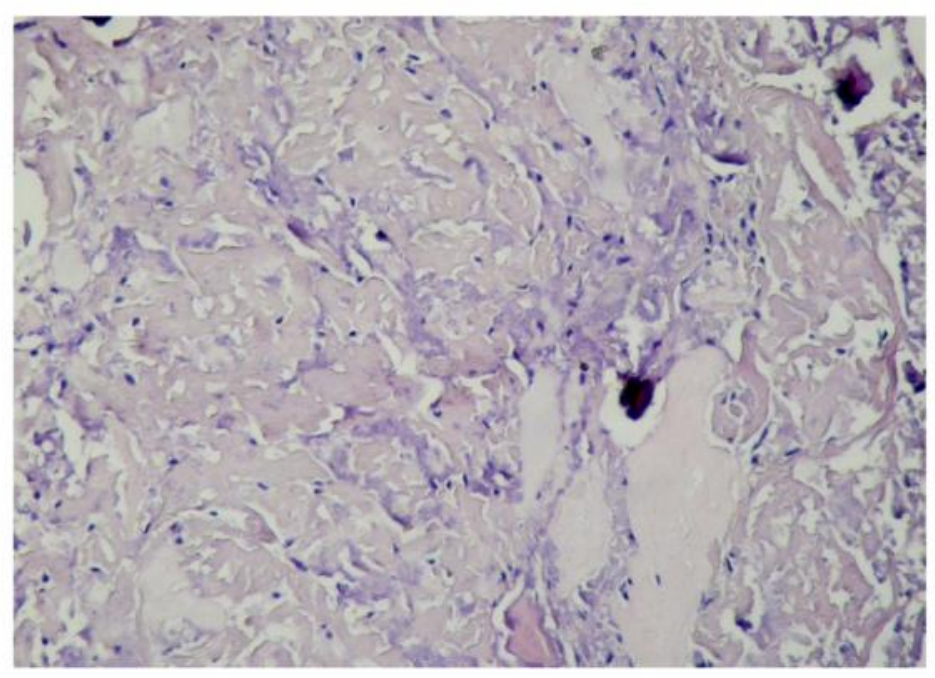

The Mantoux test was anergic and the TB Spot test was negative.

The patient posed a serious diagnostic and therapeutic problem. From the clinical perspective we were not aware what to treat - CD, TBC, sacroilitis or mesothelioma?

We started with triple TB medication and discontinuation of NSAIDs. One month later the control colonoscopy revealed cicatrices, small erosions without ulcerations, as well as sub-stenosis in the mid of colon transversum, caused by fibrotic tissue (fig.4).

Fig.4. Control colonoscopy showing diaphragm stricture.

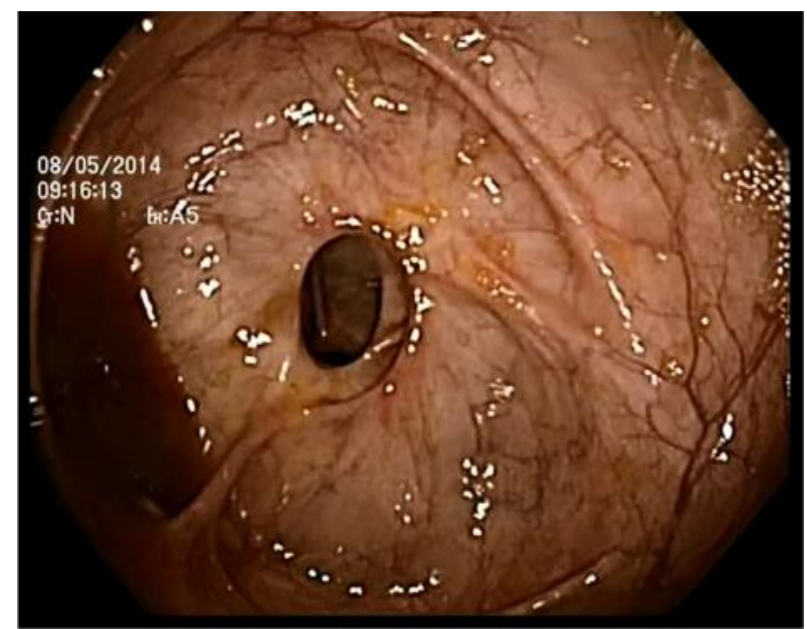

After iron infusions the haemoglobin progressively increased to normal levels. The tuberculosis treatment was without effect on the right hip and the lumbal pain, which remained severe and caused significant disability. 
Methotrexate was started 4 months after the initiation of the TB medication and a marked improvement of sacroilitis was observed. The TB medication was stopped 2 months later, while the methotrexate treatment is still ongoing. The patient remained in a good clinical condition.

Recently, a low dose IL-2 was added for treatment of mesothelioma without any complications and the methotreaxate induced lymphocytes reduction $(0.99-1.05 \mathrm{G} / \mathrm{L})$ was corrected with Interleukin-2 (Prokeukin) subcutaneously $1.2 \mathrm{MIU}-5$ days monthly $(1.44-1.65 \mathrm{G} / \mathrm{L})$

\section{Discussion}

Our case report clearly illustrates the diagnostic difficulties related to the very similar clinical, endoscopic and histological characteristics of CD with sacloilitis manifestation, intestinal TBC and NSAID-induced colopathy.

The most important clinical problem was the treatment dilemma, as the therapeutic strategies for CD and TBC are completely different. CD requires immunosuppression with steroids. In case of intestinal TBC, immunosuppression would lead to rapid disease progression and severe worsening of the patient's condition even with a possible fatal outcome. At that time, based on the patient's history of lung infection with an episode of hemoptysis and on the histological evidence of bone granulomas, as well as on one of the histological reports for intestinal tuberculosis, we initiated a triple anti tuberculosis therapy. Although the patient became persistently afebrile, no effect was registered on the right hip and the lumbar pain, which remained severe and caused significant disability.

Retrospectively, we finally consider this case to represent NSAID-induced colopathy that mimics CD and TBC. TBC seems to be far less likely due to negative TBC tests and rapid improvement of gut lesions. It is well-known that NSAID-induced injury may occur not only in the stomach and duodenum, but also in the small and large intestines $(1,8,11)$. The ulcerative lesions in the colon are less well described, although the prevalence of ileocolonic ulcers is $3.2 \%$ in chronic NSAIDs users (12). These lesions are predominantly distributed on the right and transversal parts of the colon (11). NSAID entero- and colopathy is usually asymptomatic, although some patients present with iron deficiency anemia, perforation or strictures (1). There is endoscopic evidence of progression of this condition during long term treatment with NSAID drugs from erosions to strictures. The diaphragm strictures are thin, but can reduce the size of the intestinal lumen to a pinhole (1). However, the diaphragms already formed persisted.

M. Stolte and F. Hartmann (9) recently reported a 39-year-old man who was treated as CD, with corticosteroid therapy initially and then with azathioprine. Similarly to our case, the patient was with endoscopic data for a stenosis in the transverse colon that was histologically interpreted as "consistent with CD". After surgical treatment of the stenosis the control endoscopy continued to reveal erosions and ulcers in the transverse colon. Subsequent evaluation of the histological specimens by a consultant pathologist revealed NSAID-induced colopathy.

Both cases clearly suggest that NSAID-induced colopathy is underestimated and may be a diagnostic challenge due to its pathological and endoscopic similarity with CD and TBC. 


\section{Conclusion}

Histological findings can be similar in various gut diseases with different etiology and histopathology is not sufficient for the right diagnosis. Each colonic lesion with ulceration can result in iron deficiency anemia. In many cases the patients don't mention their systemic use of NSADs, which are over-the-counter drugs. The clinical presentation of NSAID-induced colopathy is non-specific and its diagnosis can be made on colonscopy findings, including ulcerations and diaphragm-like strictures. Histological features are nonspecific but the differentiation with an inflammatory bowel disease and malignancy is of importance for the management of the patient. In our case the differential diagnosis included also intestinal tuberculosis and taking the right therapeutic decision was of great clinical importance.

\section{References}

1. Bojic D, Markovic S. Terminal ileitis is not always Crohn's disease. Annals of Gastroenterology 2011; 24: 271-275.

2. Yu H, Liu Y, Wang Y, Peng L, Li A, Zhang Y. Clinical, Endoscopic and Histological Differentiations between Crohn's Disease and Intestinal Tuberculosis. Digestion 2012; 85: 202209.

3. Epstein D, Watermeyer G, Kirsch R: Review article: the diagnosis and management of Crohn's disease in populations with high risk rates for tuberculosis. Aliment Pharmacol Ther 2007; 25: 1373-1388.

4. Almadi MA, Ghosh S, Aljebreen AM: Differentiating intestinal tuberculosis from Crohn's disease: a diagnostic challenge. Am J Gastroenterol 2009; 104: 1003-1012.

5. Ramlakan R. J.S, Govender S. Sacroiliac joint tuberculosis. Int.Orthop. 2007; 31: 121-124.

6. Hwangbo Y., Kim K.J., Park J.S., Ryu K.N., Kim N.H., Shim J., Jang J.L., Dong S.H., Kim B.H., Chang Y.W., Chang R.Sacroiliitis Is Common in Crohn's Disease Patients with Perianal or Upper Gastrointestinal Involvement. Gut Liver. 2010; 4: 338-344.

7. Lavy A, Militianu D, Eidelman S. Diseases of the intestine mimicking Crohn's disease. J Clin Gastroenterol 1992; 15 (1): 17-23.

8. Akashi M, Ando T, Hamashima T, Yoshita H, Nanjo S, Mihara $\mathrm{H}$ et al. Multiple Colon Ulcers with Typical Small Intestinal Lesions Induced by Non-Steroidal Anti-Inflammatory Drugs. Intern Med 2015; 54: 1995-1999.

9. Stolte M, Hartmann F. Misinterpretation of NSAID-induced Colopathy as Crohn's Disease. Z Gastroenterol 2010; 48: 472-475.

10. Krastev Z, Koltchakov V, Vladov N, Popov D, Milev A, Koten JW, Den Otter W. A mesothelioma that is sensitive to locally applied IL-2. Cancer Immunol Immunother. 2001; 50 (4): 226-227. 
11. Lim YJ, Yang $\mathrm{CH}$. Non-steroidal anti-inflammatory drug-induced enteropathy. Clin Endosc. 2012; 45: 138-144.

12. Amano Y, Kushiyama Y, Takahashi Y, Ishihara S, Adachi K, Kinoshita Y. Prevalence of ileocolonic ulcerative lesions in chronic NSAIDs users. Am J Gastroenterol 2004; 99: 1193.

\title{
Corresponding author:
}

\author{
Deian Jelev \\ Clinic of Gastroenterology, St. Ivan Rilsky University Hospital \\ 15, Acad. Ivan Geshov Blvd., 1431 Sofia, Bulgaria \\ Phone: +359 2952 6319, Fax: +35928510615 \\ e-mail: detjelev@gmail.com
}

